\title{
EFFECTS OF THREE-BODY INTERACTIONS ON THE STRUCTURE AND THERMODYNAMICS OF LIQUID KRYPTON
}

\author{
N. Jakse, J.M. Bomont and J.L. Bretonnet \\ Laboratoire de Théorie de la Matière Condensée, \\ Université de Metz, 1, bd F. D. Arago, CP 87811, \\ 57078 Metz Cedex 3, France
}

\begin{abstract}
Large-scale molecular dynamics simulations are performed to predict the structural and thermodynamic properties of liquid krypton using a potential energy function based on the two-body potential of Aziz and Slaman plus the triple-dipole AxilrodTeller (AT) potential. By varying the strength of the AT potential we study the influence of three-body contribution beyond the triple-dipole dispersion. It is seen that the AT potential gives an overall good description of liquid $\mathrm{Kr}$, though other contributions such as higher order three-body dispersion and exchange terms cannot be ignored.
\end{abstract}

The knowledge of interactions in noble gases remains a fundamental question that is not completely solved. Despite the simplicity of their closed-shell electronic structure, it is wellknown that a simple pair potential, though giving the essential features of the structural and thermodynamic properties, is not sufficient for a quantitative description, and many-body effects have to be taken into account 1 . Significant advances have been made when it has been demonstrated 203 that the static structure factor $S(k)$ at small wave-number, $k$, is directly related to the long range part of the effective potential between pairs of atoms. It was therefore recognized that precise measurements of $S(k)$ could provide a direct observation of the details of the interactions. During the past few years, high precision experiments 1 目, performed in the range $0.5<k<4 \mathrm{~nm}^{-1}$ using small angle neutron scattering facilities, have 
confirmed the presence of an additional interaction at large distance that can be associated to a three-body contribution at least for $\mathrm{Kr}$ 国 and $\mathrm{Xe}$ 目.

Long range interactions in noble gases arising from induced dipoles are known as the London dispersion forces 9 , and are expected to behave as $r^{-6}$, where $r$ is the interatomic distance. Quantum electromagnetic effects acting at very large separations 10 , called retardation effects, are described by the Casimir-Polder potential11, which is expected to fall off as $r^{-7}$ and to have a negligibly small influence 3 on $S(k)$. It is not surprising that a simple Lennard-Jones (12-6) potential is a satisfactory effective pair potential at first sight since it can be seen as including mean effects coming from the different dipole-dipole, multipolemultipole as well as higher order terms. However, a better understanding of the interactions lies in a careful examination of the various genuine contributions of the multipole expansion, which contains two-body $d d, d q, q q$ terms, etc., as well as irreducible three-body contributions such as $d d d, d d q, d q q, q q q$, etc., where $d$ and $q$ denote the dipole and quadrupole moment, respectively. According to Barker and Hendersont, four -and more- body terms are very small and can be neglected. The two-body potential of noble gases is often taken in the very accurate Hartree-Fock-dispersion form propounded by Aziz and Slaman12.18, while three-body dispersion effects are often represented by the $d d d$ term in the form given by Axirod and Teller 14 that represents the major contribution. The other three-body contributions mentioned above, which can be modelled by the expressions of Bell国, are known to have a small influence although it is not clear whether the agreement with the experiment could be improved when these are taken into account. At high densities, beside dispersion terms, three-body overlap contributions such as exchange effects may come into play 16 . 19.

It is tempting to consider an effective three-body potential of the Axirod-Teller 4 (AT) form

$u_{3}\left(\mathbf{r}_{i}, \mathbf{r}_{j}, \mathbf{r}_{k}\right)=\nu \frac{1+3 \cos \theta_{i} \cos \theta_{j} \cos \theta_{k}}{r_{i j}^{3} r_{i k}^{3} r_{j k}^{3}}$

to take all these three-body contributions into account by modifying the strength $\nu$ to include them empirically. In Eq. (11) $\theta_{i}, \theta_{j}$ and $\theta_{k}$ denote, respectively, the angles at vertex $i, j$ and $k$ of the triangle $(i, j, k)$ with sides $r_{i j}=\left|\mathbf{r}_{j}-\mathbf{r}_{i}\right|, r_{i k}=\left|\mathbf{r}_{k}-\mathbf{r}_{i}\right|$ and $r_{j k}=\left|\mathbf{r}_{k}-\mathbf{r}_{j}\right|$. Usually, the AT potential is designed to represent the $d d d$ contribution only with a value $\nu \equiv \nu_{d d d}=2.204 \times 10^{-26} \mathrm{~J} \mathrm{~nm}^{9}$ as prescribed by Leonard and Barker20. Such an effective three-body potential was recently used to study the liquid-vapor phase equilibria of argon21 as well as the small- $k$ part of the static structure factor of krypton in the dense liquid目. For 
instance, to interpret correctly their experimental data, Guarini et al. $\mathrm{B}$ have increased the strength $\nu$ by $65 \%$, indicating that other three-body contributions play an important role. Conversely, in a recent work 22 , we have shown that the missing contributions would have the effect of slightly reducing the strength of the AT potential. Nevertheless, these studies are not completely conclusive since calculations where carried out within the integral equations theory, in which the three-body contribution has to be treated as an approximate statedependent effective pair potential23, which is not unique.

The purpose of this article is to analyze the influence of the three-body interactions taken in the form of Eq. (11), to decide what is the effective value of the strength $\nu$ for an accurate description of liquid Kr. Three different situations are considered by varying $\nu$ : a value of $\nu=0$ that corresponds to the pure two-body potential given by Aziz and Slaman13, a value of $\nu=\nu_{d d d}$ representing only the $d d d$ contribution, and a value $\nu_{\text {eff }}=1.65 \nu_{d d d}$ which is believed to reproduce the critical parameters of $\mathrm{Kr}$ within $1 \%$. Changing the strength of the AT potential is a convenient means to measure of the departure from the $d d d$ term and, by comparison with the experiments, could provide useful information on the contribution coming from all other three-body terms that are omitted in the modelling of the interaction between Kr atoms. This work represents an extension to the liquid phase of our preceding studies on $\mathrm{Kr}$ in the gaseous phase 27.28. Thus, for a given interaction model, we carry out molecular dynamics (MD) simulations to determine $S(k)$ as well as the internal energy and the virial pressure. In this context, MD is a powerful method 24.25 since the three-body interactions can be tackled without incurring the shortcomings of the approximate integral equations. As a matter of fact, the treatment of the three-body potential (1) is not subject to arbitrariness since the resulting forces are derived in an exact manneren, as for the two-body ones.

In order to extract a meaningful structure factor $S(k)$ from the MD simulations, the pair correlation $g(r)$ is the key quantity that has to be calculated as precisely as possible. Therefore, we have performed large-scale MD in the sense that (i) a large enough simulation cell has been considered so that $g(r)$ has a sufficient spatial extension to yield a correct $S(k)$ by Fourier transform, especially at low $k$, and (ii) a large number of time steps are produced in order to get a significant part of the phase space trajectory, essential for the statistics. As three-body forces are involved, this represents a huge amount of computer time, and we have used a parallel algorithm described in some details in a previous work 29 . Simulations with the different values of $\nu$ in the AT potential have been performed in the microcanonical 
$(N V E)$ ensemble with a time step $\Delta t=5.67 \cdot 10^{-15} \mathrm{~s}$ using $N=6912$ particles in a cubic cell subject to the standard boundary conditions. The cutoff radius of the interactions is $r_{c}=2.5 r_{m}$, where $r_{m}=0.4008 \mathrm{~nm}$ is the minimum of the AS potential. For the three-body potential, this implies that triplets of particles in which two or three distances of separations are greater than $r_{c}$ are ignored in the calculation of the forces. In order to investigate the influence of $r_{c}$ for the two-body potential, a value of $4 r_{m}$ is taken in specific cases. The typical duration of the runs is $113 \mathrm{ps}$ from which 1300 independent configurations are extracted for the statistical analysis of the physical quantities. Six states of liquid Kr from the vicinity of the critical point to that of the triple point have been studied, which are those investigated by Guarini et al. $\mathrm{B}$ and by Barocchi et al.30, respectively at low- and large- $k$. These states correspond to temperature $T=199 \mathrm{~K}$ and densities $n=12.10,11.66$ and $11.31 \mathrm{~nm}^{-3}$, $T=169 \mathrm{~K}$ with $n=14.57$ and $14.22 \mathrm{~nm}^{-3}$, and $T=130 \mathrm{~K}$ with $n=16.83 \mathrm{~nm}^{-3}$.

In Fig. 1, we present the large $k$ behavior of $S(k)$ for the three different temperatures and we compare the MD curves, calculated with the three different values of $\nu$, to the experimental data of Barocchi et al. 3 . As the temperature increases and density decreases, the first sharp diffraction peak as well as the subsequent oscillations become less pronounced. This is well predicted by the MD results since a remarkable agreement with the experiments is found whatever the values of $\nu$. It can be seen that the three-body contributions have only a minor influence even if a more careful examination shows that, at low temperature, the height of the first peak is better described without the AT potential while, at high temperature, a closer agreement is obtained when it is included. At low- $k$, the three potential energy functions give rise to completely different behaviors of $S(k)$ and the best results seem to be those obtained with the $d d d$ interaction.

Let us now focus on the low- $k$ part of $S(k)$ in more details. We compare the MD curves to the recent small-angle scattering data of Guarini et al. $\mathrm{B}$ along the $T=199 \mathrm{~K}$ and $T=169 \mathrm{~K}$ isotherms in Fig. 2 (a) and (b), respectively. At the highest temperature, near the critical isotherm, a good agreement is found with the experiment at the three densities with the $d d d$ potential. Examining the influence of $\nu$ at density $n=12.10 \mathrm{~nm}^{-3}$, it appears that the AS two-body potential alone $(\nu=0)$ is not able to predict the small- $k$ part of $S(k)$ satisfactorily, while the effective AT contribution $\left(\nu=\nu_{\text {eff }}\right)$ gives rise to a structure factor which is underestimated. The chain curve corresponds to $S(k)$ obtained with the AS potential alone and a cutoff radius $r_{c}=4 r_{m}$. The influence of $r_{c}$ is seen only below $2.5 \mathrm{~nm}^{-1}$ and the curvature is slightly changed. As a result, this would have the effect of increasing the 
values of $S(k)$ in this region whatever the potential used. Taking $r_{c}=4 r_{m}$ when the threebody contribution is considered is computationally too costly and could represent a challenge for future developments of the present MD code. However, for the three-body potential, it is worth noting that, even with $r_{c}=2.5 r_{m}$, configurations in which one pair of particles of a triplet is separated up to $5 r_{m}$ are taken into account in the calculation of the forces. In addition, the AT potential given by Eq. (1) decays as $r^{-9}$ therefore taking a cutoff radius larger than $2.5 r_{m}$ seems not to be necessary. The same observation can be drawn along the $T=169 \mathrm{~K}$ isotherm, as shown in Fig 2 (b), and in this case a very good agreement with the experimental curves is seen when the $d d d$ potential is taken into account. Interesting enough, both experimental data sets of Guarini et al.8 and Barocchi et al.30, which connect to each other very well around $4 \mathrm{~nm}^{-1}$, lie between the curves corresponding to $\nu=\nu_{d d d}$ and $\nu=\nu_{e f f}$, for $k$ values in the range between 2.5 and $6.5 \mathrm{~nm}^{-1}$.

At $T=130 \mathrm{~K}$ the situation is less clear as it can be seen in Fig. 3. While the AS potential is also not sufficient to predict the small- $k$ behavior of $S(k)$, this time the best concordance with the experiment is obtained by using $\nu=\nu_{\text {eff }}$. Nevertheless, the latter effective AT contribution would have a tendency to underestimate the PVT data while the $d d d$ strength gives the best prediction. In this case, taking again a cutoff radius of $4 r_{m}$ for the two-body potential alone (chain curve) has only a negligible influence on $S(k)$, therefore our results for the two- plus three-body might be correct. It should be stressed that the amplitude of $S(k)$ is very small at such a low temperature and high density state, and the relative difference between the MD curves with $\nu_{d d d}$ and $\nu_{e f f}$ is of the same order of magnitude than the dispersion of the experimental data points, which is about $10 \%$. At this stage, we refrain from drawing any conclusion and it would be desirable to dispose of an accurate small angle scattering experiment for this thermodynamic state.

We also examine the influence of the three-body potential on the internal energy $E$ and the virial pressure $P$ gathered in Table 1 . Long-range corrections have been applied, due to the truncation of the AS and the AT potentials during the simulation at $r_{c}=2.5 r_{m}$. The corrections to the two-body part of the energy and the pressure are respectively $-0.153 n r_{m}^{3}$ and $-0.312\left(n r_{m}^{3}\right)^{2}$. For the three-body parts, the corrections are estimated numerically using the integral equation methode2s. For the energy, we obtain $0.007\left(n r_{m}^{3}\right)^{2}$ for $\nu_{d d d}$ and $0.012\left(n r_{m}^{3}\right)^{2}$ for $\nu_{e f f}$, while for the pressure we get $0.031\left(n r_{m}^{3}\right)^{3}$ for $\nu_{d d d}$ and $0.051\left(n r_{m}^{3}\right)^{3}$ for $\nu_{\text {eff }}$. These quantities are expressed in the unit of the minimum of the AS potential $\varepsilon=2.777 \cdot 10^{-21} \mathrm{~J}$. The influence of $\nu$ on the energy is moderate and does not exceed $9 \%$ 
with respect to that of the two-body potential, even with $\nu_{e f f}$. On the contrary, it has a large effect on the pressure. For the two-body potential alone, the pressure is always negative, while by including the three-body potential contribution, it becomes positive in the majority of cases. In addition, $\nu_{\text {eff }}$ yields pressure values which are too high, while the $d d d$ strength gives the best predictions, even if it is always smaller compared to the experimental data. Again, the calculated pressures with $\nu_{d d d}$ and $\nu_{e f f}$ enclose the experimental values.

Regarding the results presented above, it appears that the details of the interaction model has no significant influence on $S(k)$ at large $k$, as shown in Fig. 1. Even the AS two-body potential alone is able to reproduce the structure factor with a good degree of accuracy. For the structure factor at small scattering angle and the pressure, the three-body interactions cannot be ignored in the liquid state. Moreover, it is seen that the triple-dipole contribution gives the best agreement with the experiments and therefore represents the main three-body effect. A more precise examination shows that the structural and thermodynamic properties depart substantially from the experiments even with the model combining the AS two-body potential plus $d d d$ contribution. Indeed, discrepancies remain (i) on the small $k$ part of $S(k)$ in the range between 2.5 and $6.5 \mathrm{~nm}^{-1}$, where the calculated values are higher that the experimental ones, especially for isotherms $T=169 \mathrm{~K}$ and $T=130 \mathrm{~K}$, and (ii) on the pressure where the theoretical results are systematically below the measurements, and even remain negative for two thermodynamic states. Since the pressure varies linearly with $\nu$, as it can be seen in Table 1, better results should be obtained by increasing the value of $\nu_{\text {eff }}$ between $1.20 \nu_{d d d}$ and $1.25 \nu_{d d d}$, whatever the temperature.

We are led to the same conclusion as Guarini et al.8 that the value of $\nu$ in Eq. (1) has to be increased with respect to that of the triple-dipole $\nu_{d d d}$. However, by the light of the present MD calculations, an effective strength $\nu_{\text {eff }}=1.65 \nu_{d d d}$ seems to be too important, and we estimate that the additional three-body terms beyond the $d d d$ one should represent up to $25 \%$ of it. Now the question arises to know what is the nature of the missing contributions that will take a non negligible part in the interaction model. According to Copeland and Kestner 16 who studied liquid argon, two majors three-body potentials beyond the $d d d$ one play an important role, namely the exchange overlap and the $d d q$ dispersion acting respectively at short and long interatomic distances. Both potentials are known to have significant influence 17,31, however, while the $d d q$ potential has the same sign as the $d d d$ contribution, the exchange one has an opposite sign. Therefore, it will be of primary importance to investigate their interplay in liquid krypton and whether they improve the 
description of the interactions in liquid $\mathrm{Kr}$ when added to the $d d d$ term. It has been recently shown by van der Hoef and Madden 32 in their simulation study of liquid argon that the $d d q$ contribution on the pressure is small but not negligible and it would be interesting to extend these results in the case of $\mathrm{Kr}$, not only for the pressure but also for the structure factor.

At very small wave-number, i.e. $k<2.5 \mathrm{~nm}^{-1}$, the MD results of $S(k)$ underestimate the experimental curves as well as the PVT data, which is particularly visible along the isotherm $T=199 \mathrm{~K}$ displayed in Fig. 2 (a). As MD simulations are concerned, we are unavoidably confronted to finite size effects and this fact might be attributed mainly to two factors: the truncation of the pair-correlation functions at the half of the box size, and the use of a cutoff radius of the interactions. Therefore, the $S(k)$ calculated by Fourier transform are subject to large uncertainties and must be taken cautiously. Moreover, near the critical region, the correlation length can exceed the size of the simulation box and for this reason the present MD simulations might be not able to catch the correct behavior of $S(k)$ at $T=199 \mathrm{~K}$. As a matter of fact, as pointed out by Wilding 3 and Rover 35 , in the thermodynamic limit, critical phenomena, like the divergence of $S(0)$, are smeared out and shifted.

In conclusion, MD simulations have been carried out for $\mathrm{Kr}$ in the liquid phase for which new small angle scattering experiments were recently performed阳. This study completes preceding works on the low density and high temperature states of Kr2 29, where it was demonstrated that the Aziz and Slaman two-body potential associated to the Axirod and Teller triple-dipole contribution gives a excellent representation of interactions in the gaseous phase. Here, we have shown that the latter potential energy function predicts the essential characteristics of structural properties in liquid $\mathrm{Kr}$, even though we believe that an accurate description of $S(k)$ at low $k$ and the thermodynamic properties requires that other threebody contributions such as the dipole-dipole-quadrupole and the exchange overlap potentials are taken into account. Large scale MD simulations including these additional contributions will be performed in the near future.

The CINES (Centre Informatique National de l'Enseignement Supérieur) is gratefully acknowledged for providing us with computer time under Project No TMC1928. The authors would also like to thank Pr. R. Magli and Dr. E. Guarini for providing us with the experimental data. 


\section{REFERENCES}

1. J. A. Barker and D. Henderson, Rev. Mod. Phys. 48, 589 (1976).

2. J. E. Enderby, T Gaskell and H. H. March, Proc. Phys Soc. London. 85, 217 (1965).

3. L. Reatto and M. Tau, J. Phys.: Condens. Matter 4, 1 (1992).

4. R. Magli, F. Barocchi, P. Chieux, R. Fontana, Phys. Rev. Lett. 77, 846 (1996).

5. F. Formisano, C. J. Benmore, U. Bafile, F. Barocchi, P. A. Egelstaff, R. Magli and P. Verkerk, Phys. Rev. Lett. 79, 221 (1997).

6. F. Formisano, F. Barocchi and R. Magli, Phys. Rev. E 58, 2648 (1998).

7. C. J. Benmore, F. Formisano, R. Magli, U. Bafile, P. Verkerk, P. A. Egelstaff, and F. Barocchi, J. Phys.: Condens. Matter 11, 3091(1999).

8. E. Guarini, R. Magli, M. Tau, F. Barocchi, G. Casanova and L. Reatto, Phys. Rev. E 63, 052201 (2001).

9. F. London, Z. Phys. 63, 245 (1930).

10. E. A. Power, Eur. J. Phys., 22, 453 (2001).

11. H. B. G. Casimir and D. Polder, Phys. Rev. 73, 360 (1948).

12. R. A. Aziz and M. J. Slaman, Mol. Phys. 57, 827 (1985).

13. R. A. Aziz and M. J. Slaman, Mol. Phys. 58, 679 (1986).

14. B. M. Axilrod and E. Teller, J. Chem. Phys. 11, 299 (1943).

15. R. J. Bell, J. Phys. B 3, 731 (1970).

16. D. A. Copeland and N. R. Kestner, J. Chem. Phys. 49, 5214 (1968).

17. P. Loubeyre, Phys. Rev. B 37, 5432 (1988).

18. R. J. Sadus and J. M. Prausnitz, J. Chem. Phys. 104, 4784 (1996).

19. V. F. Lotrich and K. Szalewicz, Phys. Rev. Lett. 79, 1301 (1997).

20. P. J. Leonard and J. A. Barker, Theor. Chem. Adv. Perspect. 1, 117 (1975).

21. Y. Miyano, Fluid Phase Equilib. 95, 31 (1994). 
22. J. M. Bomont, N. Jakse and J. L. Bretonnet (to be published).

23. G. Casanova, R. J. Dulla, D. A. Johan, J. S. Rowlinson and G. Savile, Mol. Phys. 18, 589 (1970).

24. M. P. Allen and D. J. Tildesley, "Computer Simulation of liquids" (Clarendon Press 1989).

25. J. M. Haile, "Molecular Dynamics Simulation : Elementary Methods", ed. John Wiley and Sons Inc. (1992).

26. C. Hoheisel, Phys. Rev. A 23, 1998 (1981).

27. J. M. Bomont, N. Jakse and J. L. Bretonnet, Phys. Rev. B 57, 10217 (1998).

28. N. Jakse, J. M. Bomont, I. Charpentier and J. L. Bretonnet, Phys. Rev. E 62, 3671 (2000).

29. N. Jakse and I. Charpentier, Mol. Sim. 23, 293 (2000).

30. F. Barocchi, P. Chieux, R Magli, L. Reatto and M. Tau, J. Phys.: Condens. Matter 5, 42991(1993).

31. J. M. Bomont, J. L. Bretonnet and M. A. van der Hoef, J. Chem. Phys. 114, 5674 (2001).

32. M. A. van der Hoef and P. A. Madden, J. Chem. Phys. 111, 1520 (1999).

33. J. Juza and O. Sifner, Acta Technica CSAV 1, 1 (1976).

34. N. B. Wilding, J. Phys.: Condens. Matter 9, 585 (1997).

35. M. Rovere, J. Phys.: Condens. Matter 5, B193 (1993). 


\section{Captions}

Figure 1. Structure factor $S(q)$ for $T=130 \mathrm{~K}$, at $n=16.83 \mathrm{~nm}^{-3}$, for $T=169 \mathrm{~K}$, at $n=14.57 \mathrm{~nm}^{-3}$ and for $T=199 \mathrm{~K}$, at $n=12.10 \mathrm{~nm}^{-3}$ from the top to the bottom (the curves for $T=169 \mathrm{~K}$ and $T=130 \mathrm{~K}$ are shifted upwards by an amount of 1 and 2, respectively), calculated by molecular dynamics with $\nu=0$ (dashed lines) $\nu=\nu_{d d d}$ (solid lines) and $\nu=\nu_{e f f}$ (dotted lines) as described in the text. Open circles correspond to the experimental data of Ref. 30, while full circles stand for the PVT data of Ref. 33.

Figure 2. Structure factor $S(q)$ at small scattering angle for isotherm (a) $T=199 \mathrm{~K}$, at $n=12.10 \mathrm{~nm}^{-3}, n=11.66 \mathrm{~nm}^{-3}$ and $n=11.31 \mathrm{~nm}^{-3}$, and (b) $T=169 \mathrm{~K}$, at $n=14.57 \mathrm{~nm}^{-3}$. and $14.22 \mathrm{~nm}^{-3}$. The curves for $n=11.66 \mathrm{~nm}^{-3}$ and $n=11.31 \mathrm{~nm}^{-3}$ are shifted upwards by an amount of 0.5 and 1, respectively, and that of $14.22 \mathrm{~nm}^{-3}$ by an amount of 0.2. Molecular dynamics results are carried out with $\nu=0$ (dashed lines), $\nu=\nu_{d d d}$ (solid lines) and $\nu=\nu_{\text {eff }}$ (dotted lines) as described in the text. The chain curve corresponds to MD results with $\nu=0$ and a cutoff radius of $4 r_{m}$. Open circles correspond to the experimental data of Ref. 30, open triangles correspond to the experimental data of Ref. 8 and full circles stand for the PVT data of Ref. 33 .

Figure 3. Structure factor $S(q)$ at small scattering angle for $T=130 \mathrm{~K}$, at $n=16.83$ $\mathrm{nm}^{-3}$. Same captions as in Fig.1.

Table 1. Excess internal energy, $E^{\mathrm{ex}} / N \varepsilon$, and pressure, $P\left(r_{m}\right)^{3} / \varepsilon$, calculated by molecular dynamics for the different thermodynamic states considered in this work. Subscripts 2 and 3 stand respectively for the two- and two- plus three-body parts of the internal energy. $P_{\text {exp }}$ corresponds to the experimental values of Ref. 8. The numbers in brackets represent the standard deviations that affect the last decimal of the temperature, energy and pressure extracted from the simulation. 


\section{Table}

\begin{tabular}{|c|c|c|c|c|c|c|}
\hline \multicolumn{2}{|c|}{$T(\mathrm{~K}) \rho\left(\mathrm{nm}^{-3}\right) \nu / \nu_{d d d}$} & \multirow{2}{*}{$\frac{E_{2}^{\mathrm{ex}} / N \varepsilon}{-4.962(6)}$} & \multirow{2}{*}{$\frac{E_{3}^{\mathrm{ex}} / N \varepsilon}{-}$} & \multicolumn{3}{|c|}{$\operatorname{Pr}_{m}^{3} / \varepsilon P($ bar $) P_{\exp }(\mathrm{bar})$} \\
\hline \multirow[t]{3}{*}{$130(1)$} & 16.83 & & & $-0.87(4)$ & -375.4 & \\
\hline & & $1-4.953(6)$ & $-4.689(6)$ & $-0.12(4)$ & -51.8 & 0 \\
\hline & & $1.65-4.958(6)$ & $-4.522(6)$ & $0.33(4)$ & 142.4 & \\
\hline \multirow[t]{3}{*}{$169(1)$} & 14.57 & $0-4.151(7)$ & - & $-0.37(5)$ & -159.6 & \\
\hline & & $1-4.127(7)$ & $-3.938(7)$ & $0.06(4)$ & 25.9 & 61.7 \\
\hline & & $1.65-4.126(7)$ & $-3.818(7)$ & $0.30(4)$ & 129.4 & \\
\hline $169(1)$ & 14.22 & $1-4.034(7)$ & $-3.855(7)$ & $-0.05(4)$ & -21.5 & 20.1 \\
\hline \multirow[t]{3}{*}{$199(1)$} & 12.10 & $0-3.392(8)$ & - & $-0.06(4)$ & -25.9 & \\
\hline & & $1-3.358(9)$ & $-3.232(9)$ & $0.11(4)$ & 49.6 & 73.1 \\
\hline & & $1.65-3.344(8)$ & $-3.138(8)$ & $0.27(4)$ & 116.5 & \\
\hline $199(1)$ & 11.66 & $1-3.245(8)$ & $-3.127(8)$ & $0.09(4)$ & 38.8 & 55.7 \\
\hline $199(1)$ & 11.31 & $1-3.165(8)$ & $-3.053(8)$ & $0.06(4)$ & 25.9 & 46.3 \\
\hline
\end{tabular}

Table 1 


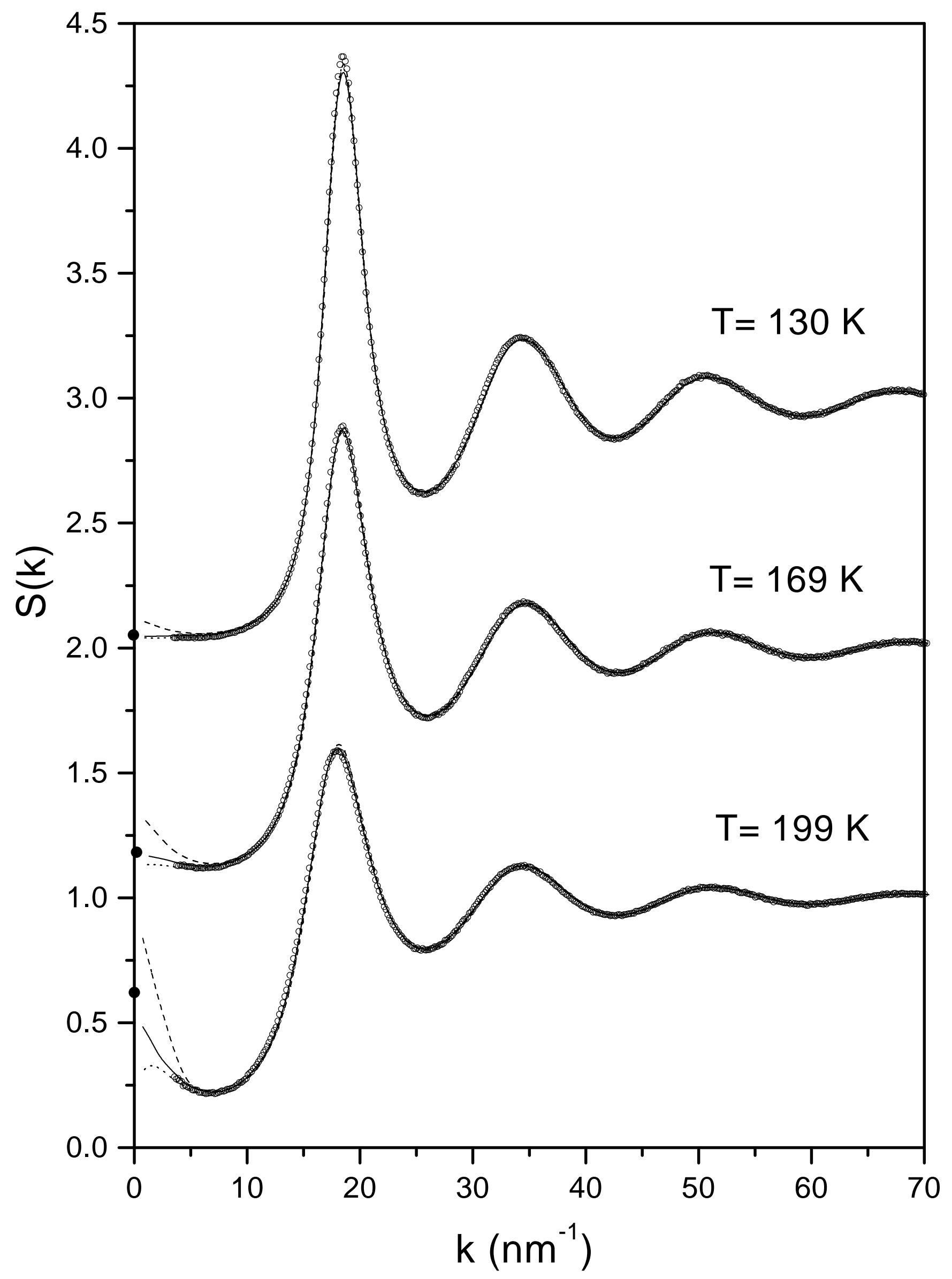



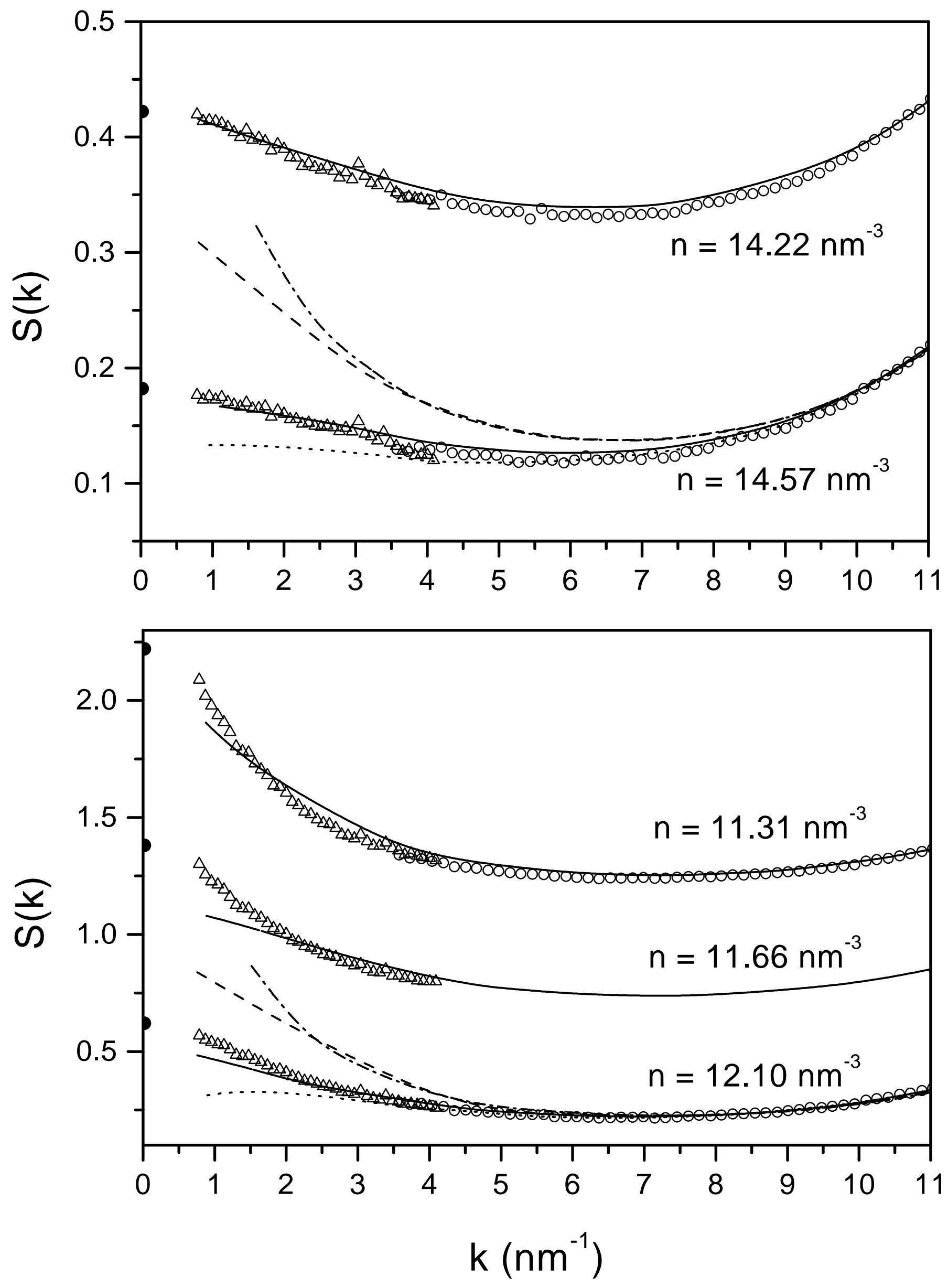


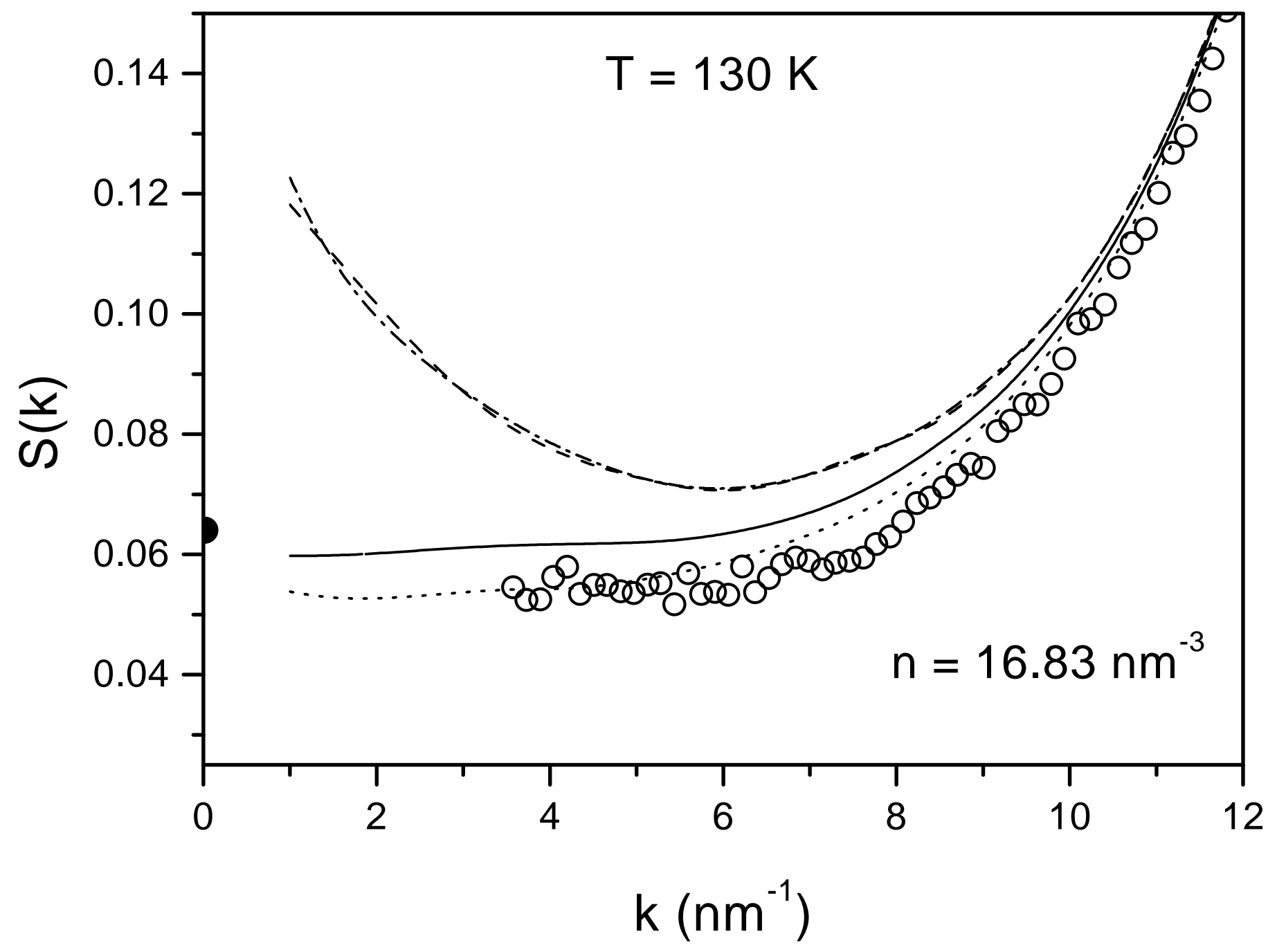

